ORIGINAL ARTICLE

\title{
Modified electrode placement must be recorded when performing 12-lead electrocardiograms
}

\author{
N I Jowett, A M Turner, A Cole, P A Jones
}

Postgrad Med J 2005;81:122-125. doi: 10.1136/pgmj.2004.021204

See end of article for authors' affiliations

\section{Correspondence to:}

Dr N I Jowett, Withybush General Hospital, Haverfordwest,

Pembrokeshire SA61 2PZ,

Wales; nigel.jowett@

pdt-tr.wales.nhs.uk

Submitted

27 February 2004

Accepted 29 March 2004
Background: Local observation has suggested that placing limb leads on the torso when recording the standard 12-lead resting electrocardiogram (ECG) has become commonplace. This non-standard modification has the important advantages of ease and speed of application, and in an emergency may be applied with minimal undressing. Limb movement artefact is also reduced. It is believed that ECGs obtained with torso electrodes are interchangeable with standard ECGs and any minor electrocardiographic variations do not affect diagnostic interpretation.

Study design: The study compared 12 -lead ECGs in 100 patients during routine electrocardiography, one being taken in the approved way and one taken with modified limb electrodes.

Results: It was found that the use of torso leads produced important amplitude and waveform changes associated with a more vertical and rightward shift of the QRS frontal axis, particularly in those with abnormal standard ECGs. Such changes generated important ECG abnormalities in $36 \%$ of patients with normal standard ECGs, suggesting "heart disease of electrocardiographic origin". In those with abnormal standard ECGs, moving the limb leads to the torso made eight possible myocardial infarcts appear and five inferior infarcts disappeared. Twelve others developed clinically important T wave or QRS frontal axis changes.

Conclusions: It is vital that ECGs should be acquired in the standard way unless there are particular reasons for not doing so, and that any modification of electrode placement must be reported on the ECG itself. Marking the ECG "torso-positioned limb leads" or "non-standard" should alert the clinician to its limitations for clinical or investigative purposes, as any lead adaptation may modify the tracing and could result in misinterpretation.
$\mathrm{T}$ he placement of limb lead electrodes on the torso is often used for continuous 12-lead electrocardiographic (ECG) monitoring, and during exercise stress testing to permit mobility. ${ }^{1-3}$ The Mason-Likar (M-L) ECG lead adaptation for stress testing relocated the wrist electrodes to the medial border of deltoid, $2 \mathrm{~cm}$ below the lower border of the clavicle in the right and left infra-clavicular fossae, and the left leg electrode to the anterior axillary line midway between the iliac crest and the costal margin. Using a similar modification for resting 12-lead electrocardiography was suggested by Takuma et al in $1994,{ }^{4}$ to permit easier application in an emergency. Limb leads were relocated to the anterior acromial region and anterior superior iliac spines bilaterally and seemed to have little overall effect on the ECG. Any changes that did occur were said to be within clinically permissible ranges. While routine use was not recommended, the appeal of this easily applied lead system has resulted in extensive use in our hospital, both in emergency and non-emergency situations. We therefore wished to test the validity of this modification in routine everyday use, as sequential ECGs were being acquired in the same patient sometimes using limb leads, and sometimes using the modified lead protocol.

\section{METHODS}

Two sequential 12-lead ECGs were carried out on adult patients on medical and surgical wards during routine electrocardiography over a four week period. ECGs were recorded in the supine position using the same Hewlett-Packard 200i Pagewriter machine and bio-adhesive diagnostic ECG skin electrodes (Biotabs, MSB Ltd, UK). One recording was made using standard limb lead placement, and the second using the modified Takuma electrode positions. Chest lead placement remained standard. The 12-lead ECGs were recorded in automatic mode, with waveform analysis being assessed by the integral analysis programme. To prevent variability of electrode placement and methodology, one cardiac technician (AC) carried out all ECG recordings. The standard ECG recordings were subsequently separated into those that were normal and those that were abnormal. Fifty paired ECGs were then taken from each group at random for analysis. Box 1 shows the

Box 1 Patient characteristics and baseline ECG findings

(A) Those with normal standard ECGs

- 29 men and 21 women

- Average age 55.7 years (range: 21-81)

(B) Those with abnormal standard ECGs

- 33 men and 17 women

- Average age: 60.1 year (range 42-89)

- Abnormalities on the initial ECG

- Inferior myocardial infarct-6

- Anterior myocardial infarct-7

- Left bundle branch block-6

- Right bundle branch block-2

- Antero-lateral ST changes-5

- Lateral ST changes -5

- Diffuse ST changes-5

- Left ventricular hypertrophy-4

- Miscellaneous abnormalities-10 
Table 1 Changes in the waveform frontal plane axis

\begin{tabular}{|c|c|c|c|c|c|c|c|c|}
\hline & \multicolumn{3}{|c|}{ Standard (S) } & \multicolumn{3}{|c|}{ Modified (M) } & \multicolumn{2}{|c|}{ Difference } \\
\hline & Mean & (SD) & Range & Mean & (SD) & Range & $(M-S)$ & p Value* \\
\hline \multicolumn{9}{|c|}{ Electrocardiographic axes in degrees for those with normal standard ECGs $(n=50$ ) } \\
\hline P axis & 43.82 & 19.24 & -17 to +76 & 55.90 & 20.06 & -26 to +88 & 12.08 & $<0.0001$ \\
\hline T axis & 29.16 & 21.45 & -22 to +81 & 53.68 & 20.01 & -34 to +83 & 24.52 & $<0.0001$ \\
\hline QRS axis & 29.02 & 28.93 & -21 to +89 & 55.96 & 25.18 & -20 to +92 & 26.94 & $<0.0001$ \\
\hline \multicolumn{9}{|c|}{ Electrocardiographic axes in degrees for those with abnormal standard ECGs ( $n=50$ ) } \\
\hline$P$ axis & 33.24 & 35.81 & -88 to +82 & 56.63 & 42.48 & -18 to +101 & 23.39 & $<0.016$ \\
\hline $\mathrm{T}$ axis & 77.37 & 77.46 & -78 to +102 & 84.08 & 79.55 & -83 to +177 & 6.71 & $<0.004$ \\
\hline QRS axis & 6.30 & 46.12 & -78 to +96 & 27.45 & 62.92 & -86 to +147 & 21.15 & $<0.002$ \\
\hline
\end{tabular}

patient characteristics. P, T, and QRS axes were calculated automatically by the ECG software and the computer analysis report was noted. The ECGs were also visually inspected (by NIJ) to check the computer analysis, and for clinically important changes without knowledge of the method of acquisition.

\section{RESULTS}

\section{Quality of the tracing}

Movement artefact in the standard leads occurred in 43 patients during limb electrode recording. This did not occur in any patient with modified electrode placement.
Changes in the waveform frontal plane axis (table 1) In comparison with the standard ECG, modified limb lead placement resulted in an average frontal plane QRS right axis shift of $26.94^{\circ}$ in those with normal resting ECGs, and in $21.15^{\circ}$ in those with abnormal resting ECGs (right axis deviation). However, there was considerable individual variation in the extent of this shift, being more pronounced in those who had an abnormal initial ECG. Similar wide variation of the $\mathrm{P}$ and $\mathrm{T}$ wave axis was also seen. Consequent to this change in axis, there was reduction in the $\mathrm{R}$ wave amplitude in leads I and aVL with increased $\mathrm{R}$ wave amplitude in the inferior leads (II, III, and aVF). Any inferior Q waves became less prominent, and sometimes insignificant. S wave

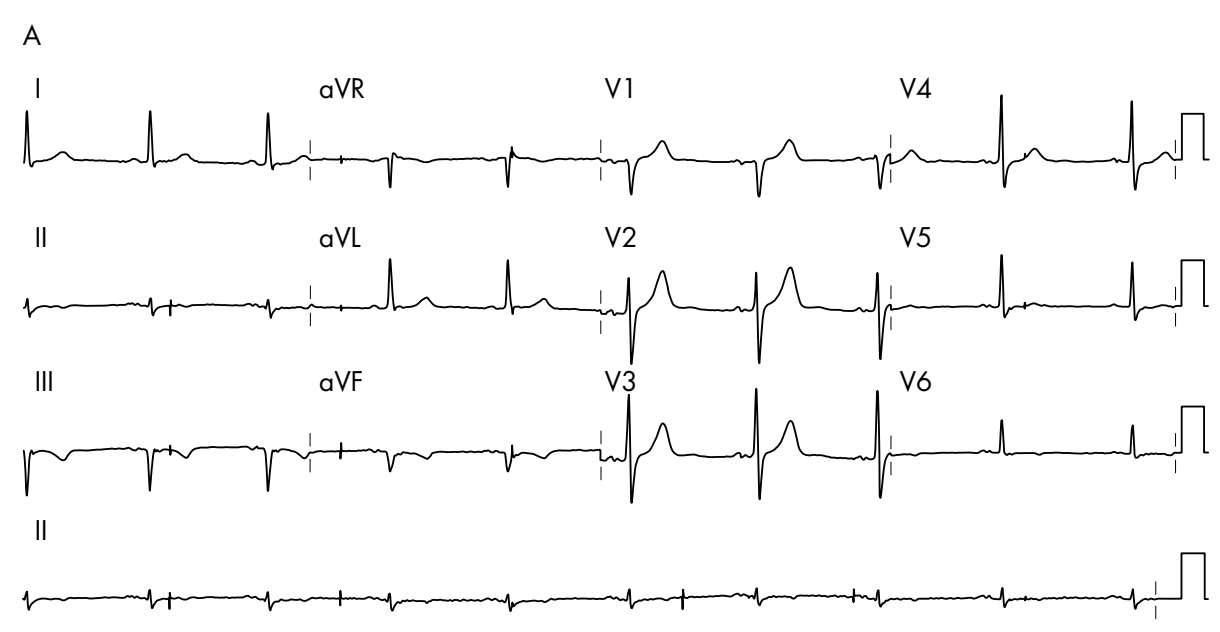

Figure 1 Inferior myocardial infarction disappears on moving limb leads from legs to torso.

B

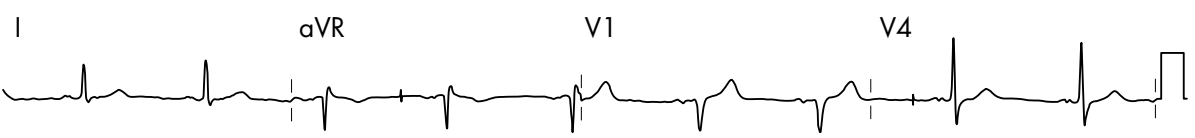

$\begin{array}{llll}\| & \mathrm{aVL} & \mathrm{V} 2 & \mathrm{~V} 5\end{array}$
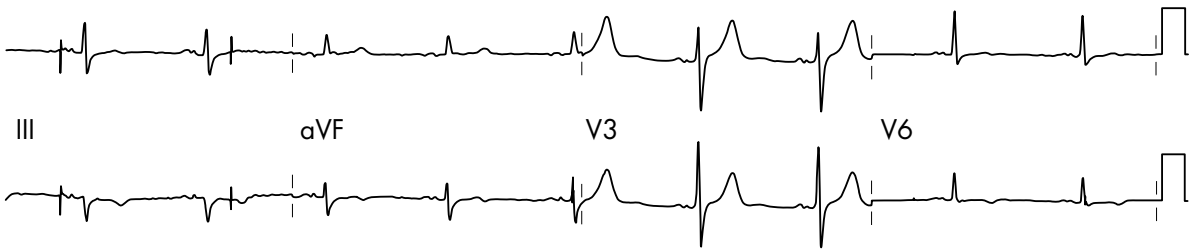

II

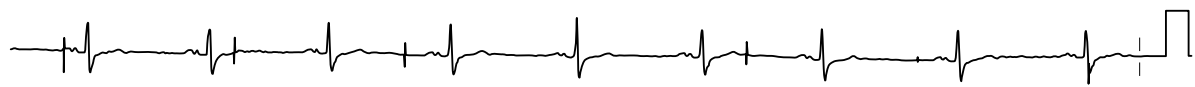


A

I $\quad$ aVR

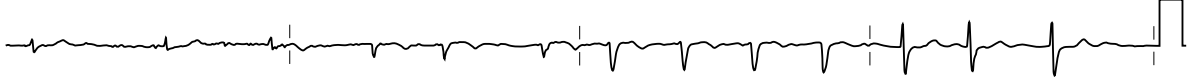

$\begin{array}{llll}\| & \text { aVL } & \text { V2 } & \text { V5 }\end{array}$

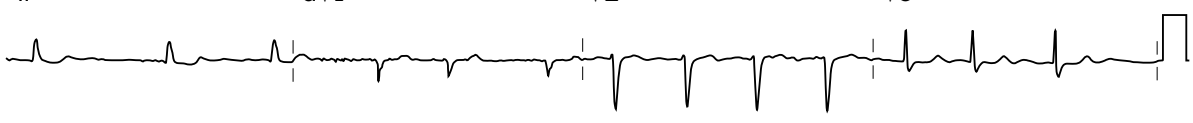

$\begin{array}{llll}\text { III } & \text { aVF } & \text { V3 } & \text { V6 }\end{array}$

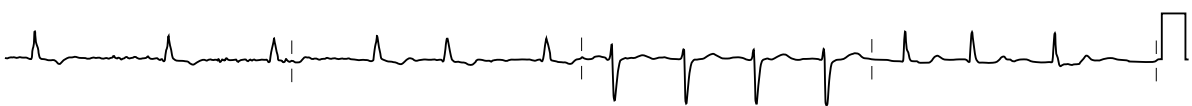

II

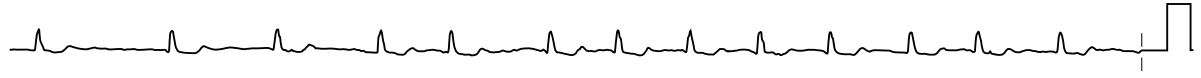

B

$\begin{array}{llll}\text { I } & \text { aVR } & \text { V1 } & \text { V4 }\end{array}$

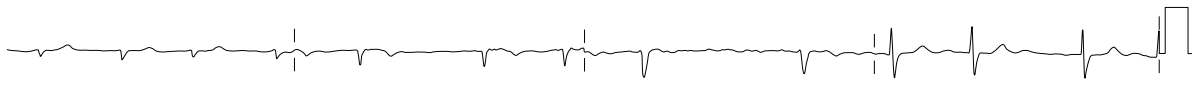

$\begin{array}{llll}\| & \mathrm{aVL} & \mathrm{V} 2 & \mathrm{~V} 5\end{array}$
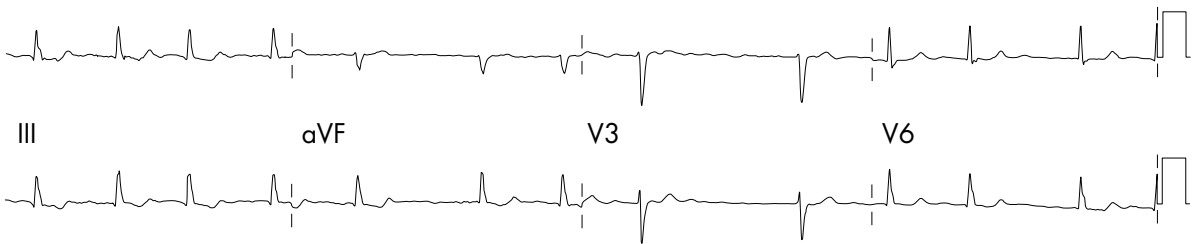

॥

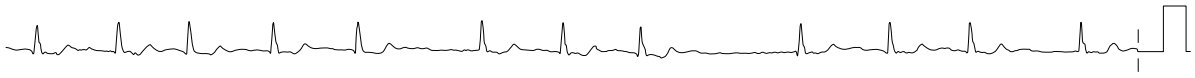

amplitude increased in leads II and aVF and decreased in III. $\mathrm{P}$ and $\mathrm{T}$ wave amplitude changes mainly mirrored those seen in $\mathrm{R}$ wave amplitude. $\mathrm{T}$ waves became flattened or bi-phasic in aVL. Where apparent, ST slope increased in the inferior leads, and decreased in aVL, changes suggesting acute inferior injury.

\section{Changes in the chest leads}

Although the central terminal should have an altered potential, there seemed to be little changes in the chest $(\mathrm{V})$ lead recordings, but where apparent, there was a decrease in the R waves in V1-2 and the S waves in V5-6. Resultant QS patterns in V1-V2 in 4 cases suggested anterior infarction.

\section{Computer diagnosis}

Eighteen ECGs had a computer reported abnormality with the modified ECG leads when the standard ECG was normal. These were:

- High frontal QRS voltage defined as $\mathrm{R}$ in $\mathrm{II}>1.5 \mathrm{mV}$ or $\mathrm{R}$ in $\mathrm{aVL}>1.2 \mathrm{mV}(11)$

- Non-specific inferior T wave abnormalities (2)

- ST elevation in inferior leads

- Non-specific anterior T wave abnormalities

- Lateral ST elevation

- Right axis deviation $\left(>105^{\circ}\right)$

- Probable left ventricular hypertrophy (LVH)
Figure 2 Lateral myocardial infarction appears on moving limb leads from legs to torso.
In those with abnormal standard ECGs, there was diagnostic agreement in 25 of the 50 paired ECGs. However, moving the limb leads to the torso changed the baseline ECG to make eight possible myocardial infarcts appear (four anterior, three inferior, one lateral), and five inferior infarcts disappeared. Examples are shown in figures 1 and 2. Other consequences were loss of inferior and lateral $\mathrm{T}$ wave changes (2) and development of infero-lateral $\mathrm{T}$ wave changes (5). Three patients developed abnormal right axis deviation $\left(>+105^{\circ}\right)$. Two patients with left axis deviation $\left(>-30^{\circ}\right)$ produced a normal ECG when torso leads were used.

\section{DISCUSSION}

Precise recording of the standard 12-lead ECG is technically exacting and time consuming. Placing limb leads on the torso has the major advantages of ease and speed of application, and in an emergency may be applied with minimal undressing. Limb movement artefact is also reduced, or prevented as seen in our series. It has been asserted that ECGs obtained with torso electrodes are interchangeable with standard ECGs and any minor variations do not affect diagnostic interpretation. ${ }^{1-3}$ This was not true in our patients, where 18 normal people would develop what Marriott termed "heart disease of electrocardiographic origin". ${ }^{6}$ Such abnormalities may generate unnecessary investigation and worry. We found that limb lead modification produced important amplitude and waveform changes associated with a more vertical and rightward shift of the QRS frontal axis, 


\section{Learning points}

- Placing limb leads on the torso when recording 12lead ECGs is common and seldom reported

- Modified leads are not always placed in the same positions on the torso

- Torso modifications of limb leads are non-standard and can affect the ECG trace

- The most frequent changes are produced by right axis deviation

- Such changes may obscure or create changes of myocardial infarction

- Other important T wave or frontal QRS axis changes may occur

- Abnormal ECGs may be produced in normal people

- ECGs should be acquired using the standard lead positions unless there are exceptional circumstances

- Any electrode modification must be marked on the ECG

- If marked "non-standard" or "limb leads modified", 12-lead ECGs have limitations for diagnostic purposes

particularly in those with abnormal standard ECGs. Other investigators have noted similar ECG distortions when limb leads are repositioned on the body, ${ }^{7-8}$ although modifications of the limb leads in these studies were not uniform. This is important, because placing of the left ankle electrode on different parts of the torso can result in very different ECG patterns, all of which may be at variance from the standard ECG.' Mason and Likar believed that the left leg electrode could be "varied by a few centimetres in any direction without significant effect on the ECG". ${ }^{1}$ However, the M-L modification has been found to create right axis shifts of up to $60^{\circ}$, producing or obscuring evidence of previous infarction. ${ }^{8}$ An average right axis deviation of $48^{\circ}$ was noted when the leg electrodes were placed much closer to the heart (above the right and left sub-costal regions), a modification that could also make posterior and inferior infarcts disappear, and lateral infarcts appear. ${ }^{10}$ Least interference seems to result when the modified left leg electrode is placed close to the anterior superior iliac spine, ${ }^{479}$ although our finding of a mean right axis change of $24^{\circ}$ is greater than in other reports. Different study groups and interpersonal variation may explain such inconsistencies. For example, increasing age and obesity may associate with a change in axis to the left. Any rightward shift caused by torso leads may return it to within normal limits. Takuma ${ }^{4}$ recorded the two ECGs simultaneously to allow for differences caused by respiration, but it is unlikely that this is a major confounder. While the amplitude of the Q wave in lead III may be reduced by deep inspiration, this is not a usual consideration in routine electrocardiography.
The basis for Einthoven's (equilateral) triangle was positioning the electrodes of the three standard leads as far away from the heart as possible to ensure equidistance of the recording points, although according to the only specific reference to standard limb lead placement we could find:

"the (limb) electrodes may be placed on any part of the arms or the left leg as long as they are below the shoulders in the former and below the inguinal fold anteriorly and the gluteal fold posteriorly in the latter. Any other placement necessary by deformed or missing extremities must be noted on the record. ${ }^{\prime \prime 11}$

We suggest that any modification of electrode placement must be reported on the ECG itself, regardless of the reason, as any adaptation may modify the tracing and could result in misinterpretation. All ECGs should be acquired using the standard lead positions unless there are exceptional circumstances. Marking the ECG "torso-positioned limb leads" or "non-standard" should alert the clinician to its limitations for clinical or investigative purposes. However, the precise positioning of the electrodes should also be noted, as each modification will produce its own unique recording pattern, making comparisons between variants as prone to error as comparisons with the standard ECG.

\section{Authors' affiliations}

N I Jowett, A M Turner, A Cole, P A Jones, Department of Cardiovascular Medicine, Pembrokeshire and Derwen NHS Trust, Wales

Funding: the study received financial support from the Dyfed Research and Development Consortium.

Conflicts of interest: none declared.

\section{REFERENCES}

1 Mason RE, Likar I. A new system of multiple-lead exercise electrocardiography. Am Heart J 1966;71:196-205.

2 Edenbrandt L, Pahlm O, Sornmo L. An accurate exercise lead system for bicycle ergometer tests. Eur Heart J 1989;10:268-72

3 Fesmire FM, Smith EE. Continuous 12-lead electrocardiograph monitoring in the emergency department. Am J Emerg Med 1993;11:54-60.

4 Takuma K, Hori S, Sasaki J, et al. An alternative limb lead system for electrocardiography in emergency patients. Am J Emerg Med 1995; 13:514-17

5 Diamond D, Griffith DH, Greenberg ML, et al. Torso mounted electrocardiographic electrodes for routine clinical electrocardiography. Journal of Electrophysiology 1979;12:403-6.

6 Marriott H. Practical electrocardiography. 6th ed. Baltimore: Williams and Wilkins, 1977:266.

7 Rautahariu PM, Prineas RJ, Crow RS, et al. The effect of modified limb electrode positions on electrocardiographic wave amplitudes. Journal of Electrocardiography 1980;13:109-13.

8 Kleiner JP, Nelson WP, Boland MJ. The 12-lead electrocardiogram in exercise testing. A misleading baseline? Arch Intern Med 1978;138:1572-3.

9 Papouchado M, Walker PR, James MA, et al. Fundamental differences between the standard 12-lead electrocardiograph and modified (Mason-Likar) exercise lead system. Eur Heart J 1987;8:725-33.

10 Sevilla DC, Dohrmann ML, Sornelofski CA, et al. Invalidation of the resting electrocardiogram obtained via exercise electrode sites as a standard 12-lead recording. Am J Cardiol 1989;63:35-9

11 Pipberger HV, Arzbaecher RC, Berson AS, et al. Recommendations for standardization of leads and of specifications for instruments in electrocardiography and vectorcardiography. Circulation 1975;52:11-31. 